\title{
Prognostic Impact of Immunonutritional Status Changes During Preoperative Chemoradiation in Patients With Rectal Cancer
}

\author{
Yong Joon Lee, Woo Ram Kim¹, Jeonghee Han, Yoon Dae Han, Min Soo Cho, Hyuk Hur, Kang Young Lee, \\ Nam Kyu Kim, Byung Soh Min \\ Department of Surgery, Severance Hospital, Yonsei University College of Medicine, Seoul; ${ }^{1}$ Department of Surgery, CHA Bundang Medical \\ Center, CHA University, Seongnam, Korea
}

Purpose: Previous studies have demonstrated the prognostic impact of the prognostic nutritional index (PNI), a proposed indicator of immunonutritional statuses of surgical patients, on patients with various gastrointestinal cancers. Although the prognostic impact of the PNI on patients with colorectal cancer has been well established, its value has not been studied in patients treated with preoperative chemoradiation (pCRT). This study aimed to evaluate the prognostic impact of PNI on patients receiving pCRT for locally advanced rectal cancer (LARC).

Methods: Patients with LARC who underwent curative pCRT followed by surgical resection were enrolled. The PNI was measured in all patients before and after pCRT, and the difference in values was calculated as the PNI difference (dPNI). Patients were classified according to dPNI $(<5,5-10$, and $>10)$. Clinicopathologic parameters and long-term oncologic outcomes were assessed according to dPNI classification.

Results: No significant intergroup differences were observed in clinicopathologic parameters such as age, histologic grade, tumor location, tumor-node-metastasis stage, and postoperative complications. Approximately $53 \%$ of the patients had a mild dPNI $(<5)$; only $15 \%$ had a high dPNI $(>10)$. Univariate and multivariate analyses identified the dPNI as an independent prognostic factor for disease-free status ( $\mathrm{P}<0.01$; hazard ratio [HR], 2.792; 95\% confidence interval [CI], 1.577 4.942 ) and for cancer-specific survival ( $\mathrm{P}=0.012$; HR, 2.469; 95\%CI, 1.225-4.978).

Conclusion: The dPNI is predictive of long-term outcomes in pCRT-treated patients with LARC. Further prospective studies should investigate whether immune-nutritional status correction during pCRT would improve oncologic outcomes.

Keywords: Rectal neoplasms; Preoperative chemoradiation; Prognostic nutritional index; Immunonutritional status

\section{INTRODUCTION}

In recent decades, many advances have been made in the treatment of locally advanced rectal cancer (LARC), including multi-

Received: September 23, 2016 - Accepted: October 17, 2016

Correspondence to: Byung Soh Min, M.D.

Department of Surgery, Severance Hospital, Yonsei University College of Medicine, 50-1 Yonsei-ro, Seodaemun-gu, Seoul 03722, Korea Tel: +82-2-2228-2100, Fax: +82-2-313-8289, E-mail: bsmin@yuhs.ac

(C) 2016 The Korean Society of Coloproctology

This is an open-access article distributed under the terms of the Creative Commons Attribution NonCommercial License (http://creativecommons.org/licenses/by-nc/4.0) which permits unrestricted noncommercial use, distribution, and reproduction in any medium, provided the original work is properly cited. disciplinary approaches involving surgery, radiotherapy, chemotherapy, and biologic agents. In particular, the combination of neoadjuvant chemoradiation therapy (CRT) and subsequent surgery improves the likelihood of sphincter preservation, decreases the locoregional recurrence rate, and improves the long-term survival rate $[1,2]$.

Cancer patients frequently develop malnutrition, a significant risk factor with regard to treatment-related morbidity and mortality, as well as treatment failure [3-7]. Accordingly, attempts to accurately evaluate and vigorously enhance the nutritional statuses of malnourished patients are ongoing [4, 7-9]. In particular, patients with LARC tend to have a worse nutritional status consequent to a longer duration of illness and obstructive symptoms. 
Furthermore, during neoadjuvant CRT, patients may develop aggravated malnutrition subsequent to high-grade gastrointestinal (GI) toxicities induced by CRT. In this context, nutritional status evaluations to detect malnourished patients might be an important step toward improving patient outcomes, and such monitoring should be performed on a serial follow-up basis.

The prognostic nutritional index (PNI), which is based on the peripheral blood serum albumin level and total lymphocyte count, was first suggested as an indicator of the immunonutritional statuses of surgical patients [10]. Notably, this index was found to correlate with postoperative morbidity and mortality. The efficiency, simplicity, and convenience of the PNI have led to its wide use as a tool for assessing the preoperative conditions and predicting the surgical risks of patients with GI malignancies. Recent investigations have also revealed that the PNI reflects the immune statuses of cancer patients and can serve as an independent prognostic factor for patients with various cancers [11-13].

Recent studies have shown that the preoperative PNI is a useful predictor of postoperative complications and survival in patients with colorectal cancer [14-17]. However, previous studies have addressed neither the efficiency of the PNI as a prognostic marker for patients with LARC nor changes in the immunonutritional statuses of patients during neoadjuvant CRT. Therefore, this study aimed to determine the relationship between the immunonutritional status and prognoses in patients with LARC who were treated with neoadjuvant CRT followed by surgery, with a particular focus on changes in the immunonutritional status after preoperative chemoradiation (pCRT) and the impact of this parameter on oncologic outcomes.

\section{METHODS}

\section{Patients}

A total of 341 patients with LARC who underwent preoperative CRT and subsequent surgery between January 2005 and December 2010 were identified from the Colorectal Cancer Registry of our institution, a prospective database. We retrospectively reviewed these patients' medical records and excluded patients whose cases did not meet the following eligibility criteria: (1) adenocarcinoma arising from the rectum, (2) completion of preoperative CRT followed by surgery with curative intent, (3) American Joint Committee on Cancer (AJCC) stage 0 (complete pathologic response) to stage III, and (4) available follow-up data. The following additional exclusion criteria were applied: (1) distant metastasis or surgery with palliative aim, (2) any palliative surgery (e.g., ileostomy) because of obstruction before preoperative CRT, and (3) missing clinical data because neoadjuvant CRT was administered at other institutions or unclear laboratory results were recorded in the database. After these criteria had been applied, 80 of the initial 341 patients were excluded (Fig. 1). This study was approved by the Institutional Review Board and informed consent was waived.

\section{Patients diagnosed as having rectal cancer suregery after preoperative $\mathrm{CRT} \pm$ Adjuvant $\mathrm{CTx}$ (2005.01.01-2010.12.31)}

Exclusion criteria

33 Distant mets or palliative surpery

6 Palliative stomy before CRT

41 Missing data

A total of 80 patients were excluded

261 Patients

Fig. 1. Overall study design and overview of the patient population. CRT, chemoradiation therapy; CTx, chemotherapy; mets, metastasis.

\section{Clinicopathologic characteristics}

Patients' clinicopathologic characteristics were collected retrospectively from medical records and evaluated as prognostic factors. Collected characteristics included age, sex, preoperative body mass index (BMI), histopathological tumor characteristics (differentiation), distance from anal verge, TNM stage, comorbidity, postoperative complications, operation time, and use of adjuvant therapy. The 7th AJCC staging system was used to determine the TNM stages of the colorectal cancers.

Postoperative complications were defined according to the Clavien-Dindo classification [18]. Comorbidity was classified according to the American Society of Anesthesiologists physical status classification system. Additionally, the following laboratory data were collected from peripheral blood analyses: carcinoembryonic antigen (CEA) and albumin levels, lymphocyte percentage, and lymphocyte count. The PNI was subsequently calculated using the following formula: $10 \times$ serum albumin $(\mathrm{g} / \mathrm{dL})+0.005 \times$ total lymphocyte count $\left(\right.$ per $\left.\mathrm{mm}^{3}\right)$ [19]. The PNI values were determined at 2 points: before preoperative CRT (pre-CRT PNI), and 4 weeks after the completion of preoperative CRT but before surgery (post-CRT PNI). The PNI difference (dPNI) was calculated using the following formula: pre-CRT PNI - post-CRT PNI.

\section{Preoperative chemoradiation and surgery}

Indications for preoperative CRT included $\mathrm{T} 3$ or $\mathrm{T} 4$ disease or a positive lymph node status based on clinical and radiologic examinations. Preoperative CRT comprised 5-fluorouracil (5-FU)based chemotherapy and pelvic irradiation (45-50 Gy in 25 fractions over 5 weeks). Chemotherapy was administered as either a continuous intravenous infusion of 5-FU and leucovorin or oral capecitabine. Curative resection comprising total or partial mesorectal excision according to the tumor level was performed 6-8 weeks after the completion of pCRT. Decisions regarding the performance of a diverting loop ileostomy were made by the operat- 
ing surgeon in consideration of the patient's risk factors. Adjuvant chemotherapy was recommended for all patients within 4-6 weeks after surgery. A pathologic examination, tumor regression grade (TRG) and standardized pathologic examination report were completed for every case. The standard form required mandatory reporting of tumor differentiation, depth of tumor penetration, lymph node metastasis, circumferential resection margin (CRM), lymphovascular invasion, and TRG, as suggested by Mandard et al. [20]. CRM involvement was defined as the presence of tumor cells from the outermost margin of the lesion to the proper mesorectal fascia or as a maximum distance of $<1 \mathrm{~mm}$ between the tumor and proper rectal fascia.

\section{Follow-up policy}

Patients underwent routine check-ups every 3 months for up to 2 years and then every 6 months for up to 3 additional years (i.e., 5 th postoperative year). A physical examination and laboratory testing, including serum CEA levels, were performed at each visit. Imaging screens, including an abdominopelvic computed tomography (CT) scan, were performed every 6 months; chest CT was performed annually. Nonroutine magnetic resonance imaging and positron emission tomography-CT were performed at the clinician's discretion. Endoscopic examinations were performed within the first postoperative year and then every $3-4$ years according to the findings.

\section{Statistical analysis}

Categorical data are presented as frequencies or percentages. Continuous data are presented as means with standard deviations or medians with ranges. Intergroup differences were analyzed using the chi-square test, Student t-test, and Mann-Whitney U-test. The Kaplan-Meier method was used to analyze survival, and the resulting curves were compared using the log-rank test. Cox proportional hazard model was used for the univariate and the multivariate analyses of the prognostic factors. All variables that were significant in the univariate analysis were included in the multivariate analysis. A P-value $<0.05$ was considered significant, and confidence intervals (CIs) were calculated at the 95th percentile level. IBM SPSS Statistics ver. 20.0 (IBM Co., Armonk, NY, USA) was used for the statistical analysis.

\section{RESULTS}

\section{Grouping of patients according to PNI differences}

A total of 261 consecutive patients were enrolled in this study. $\mathrm{Pa}-$ tients were divided into the following 3 groups according to dPNI values (defined above): dPNI-low, dPNI < 5; dPNI-moderate, dPNI of 5-10; and dPNI-high, dPNI > 10 (Fig. 2).

\section{Clinicopathologic characteristics and short-term outcomes}

The clinicopathologic characteristics and short-term outcomes of the three groups were evaluated (Table 1). The median follow-up

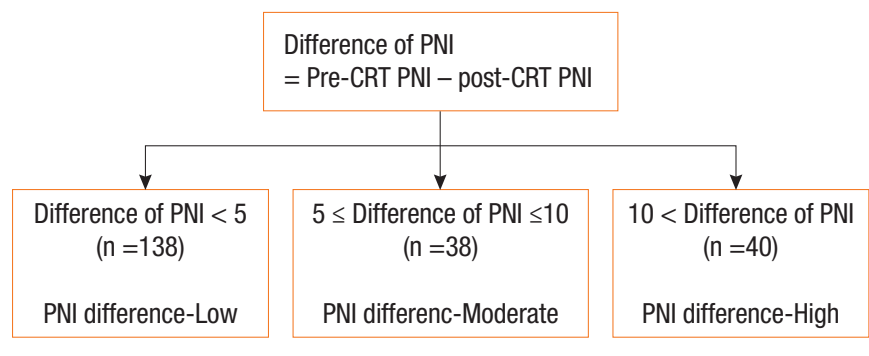

Fig. 2. Subgroup analysis according to PNI difference values. PNI, prognostic nutritional index; CRT, chemoradiation therapy.

duration was 48 months (range, 1-91 months). Approximately $88 \%$ of the patients received adjuvant chemotherapy. Except for preoperative BMI, no statistically significant intergroup differences were observed in terms of patient characteristics or tumor and operative parameters. Additionally, no significant differences were observed in short-term operative outcomes, including the incidence of high-grade (Clavien-Dindo grade III-IV) perioperative complications and 30-day mortality rate. The proportion of low rectal cancers (tumor location within $5 \mathrm{~cm}$ from the anal verge) tended to be higher in the dPNI-high group, but this difference was not statistically significant.

\section{Long-term oncologic outcomes}

Kaplan-Meier survival curves demonstrated that both the diseasefree survival (DFS) and the cancer-specific survival (CSS) outcomes were worse in the dPNI-high group relative to the other groups (DFS: $\mathrm{P}<0.001$, Fig. 1; CSS: $\mathrm{P}=0.02$, Figs. 3, 4). The univariate and the multivariate analyses of the DFS demonstrated that the ypT stage, ypN stage, and dPNI were independent predictors for DFS (Table 2). Furthermore, histology, ypT stage, and dPNI were identified as significant prognostic factors for CSS (Table 3).

\section{DISCUSSION}

Since its initial introduction by Onodera et al. in 1986 [19], the PNI has been identified as an independent prognostic factor in various types of cancer [11-17, 19]. Furthermore, in recent studies of patients with colorectal cancer, the PNI was found to be associated not only with surgical morbidity, but also with long-term oncologic outcomes [14-16]. To the best of our knowledge, however, no studies have evaluated the prognostic significance of the PNI in patients with LARC. Furthermore, this study is the first to introduce the $\mathrm{dPNI}$, a parameter that demonstrates the significance of a serial change of the PNI rather than of a single value measured at a specific time point.

More than half of the enrolled patients were classified into the dPNI-low group, and the PNI values of approximately a third of the patients in this group actually increased during the study. However, long-term oncologic outcomes did not significantly dif- 
Table 1. Patient and tumor characteristics and short-term outcomes $(n=261)$

\begin{tabular}{|c|c|c|c|c|}
\hline \multirow{2}{*}{ Variable } & \multicolumn{3}{|c|}{ dPNI } & \multirow{2}{*}{ P-valu } \\
\hline & $<5(n=138)$ & $5-10(n=83)$ & $>10(n=40)$ & \\
\hline Follow-up (mo) & $48(6-88)$ & $48(3-87)$ & $51(1-91)$ & 0.780 \\
\hline Age (yr) & $59.59 \pm 11.50$ & $57.50 \pm 10.12$ & $56.72 \pm 12.08$ & 0.223 \\
\hline Sex & & & & 0.668 \\
\hline Male & $99(71.7)$ & $55(66.3)$ & $27(67.5)$ & \\
\hline Female & $39(28.3)$ & $28(33.7)$ & $13(32.5)$ & \\
\hline Pre-CRT BMI $\left(\mathrm{kg} / \mathrm{m}^{2}\right)$ & $22.56 \pm 2.81$ & $22.45 \pm 2.88$ & $23.73 \pm 2.93$ & 0.047 \\
\hline Preoperative serum CEA (ng/mL) & & & & 0.301 \\
\hline$<5$ & $99(68.1)$ & $48(57.8)$ & $26(65.0)$ & \\
\hline$\geq 5$ & $44(31.9)$ & $35(32.2)$ & $14(35.0)$ & \\
\hline Differentiation & & & & 0.217 \\
\hline Well + moderate & $123(89.1)$ & $75(90.4)$ & $32(80.0)$ & \\
\hline Poor + mucinous & $15(10.9)$ & $8(9.6)$ & $8(20.0)$ & \\
\hline Distance from AV (cm) & & & & 0.070 \\
\hline Low $($ AV $<5)$ & $67(48.6)$ & $51(61.4)$ & $26(65.0)$ & \\
\hline Mid/upper (AV $\geq 5)$ & $71(51.4)$ & $32(38.6)$ & $14(35.0)$ & \\
\hline ASA physical status (\%) & & & & 0.870 \\
\hline$<|l|$ & 135 & 80 & 39 & \\
\hline$\geq|l|$ & 3 & 3 & 1 & \\
\hline ypT stage & & & & 0.796 \\
\hline $0 / 1 / 2$ & $56(40.6)$ & $37(44.6)$ & $18(45.0)$ & \\
\hline $3 / 4$ & $82(59.4)$ & $46(55.4)$ & $22(55.0)$ & \\
\hline ypN stage & & & & 0.152 \\
\hline 0 & $103(74.6)$ & $53(63.9)$ & $31(77.5)$ & \\
\hline $1 / 2$ & $35(25.4)$ & $30(36.1)$ & $9(22.5)$ & \\
\hline Adjuvant therapy & & & & 0.744 \\
\hline Yes & $120(87.0)$ & $75(90.4)$ & $35(87.5)$ & \\
\hline No & $18(13.0)$ & $8(9.6)$ & $5(12.5)$ & \\
\hline Operation type & & & & 0.202 \\
\hline Open & $63(45.7)$ & $45(54.2)$ & $60(60.0)$ & \\
\hline MIS & $75(54.3)$ & $38(45.8)$ & $40(40.0)$ & \\
\hline Conversion rate (\%) & $4(2.9)$ & $3(3.6)$ & $0(0.0)$ & 0.765 \\
\hline Complication (Clavien-Dindo classification) & & & & 0.480 \\
\hline$<111$ & $127(92.0)$ & $77(92.8)$ & $39(97.5)$ & \\
\hline$\geq|| \mid$ & $11(8.0)$ & $6(7.2)$ & $1(2.5)$ & \\
\hline 30-Day mortality (\%) & 0 & 0 & 1 & NS \\
\hline
\end{tabular}

Values are presented as median (range), mean \pm standard deviaion, or number (\%).

dPNI, prognostic nutritional index difference; CRT, chemoradiation therapy; BMI, body mass index; CEA, carcinoembryonic antigen; AV, anal verge; ASA, American Society of Anesthesiologists; MIS, minimal invasive surgery (laparoscopic or robotic); NS, not significant.

dPNI was calculated as follows: pre-CRT PNI - post-CRT PNI.

fer between patients in this group with an increased PNI and those with a decreased PNI (data not shown). Accordingly, we categorized both types of patients into a single dPNI-low category. In our study, the dPNI was clearly associated with long-term oncologic outcomes (DFS, CSS). To date, however, no clear explanation has been given for the correlation between the PNI and prognostic significance. Malnutrition is a known risk factor for postoperative complications in surgical patients $[4,6,7]$. Some 


\section{$\begin{aligned} \text { Annals of } & \text { Patients With Rectal } \\ \text { Coloproctology } & \text { Yong Joon Lee, et al. }\end{aligned}$

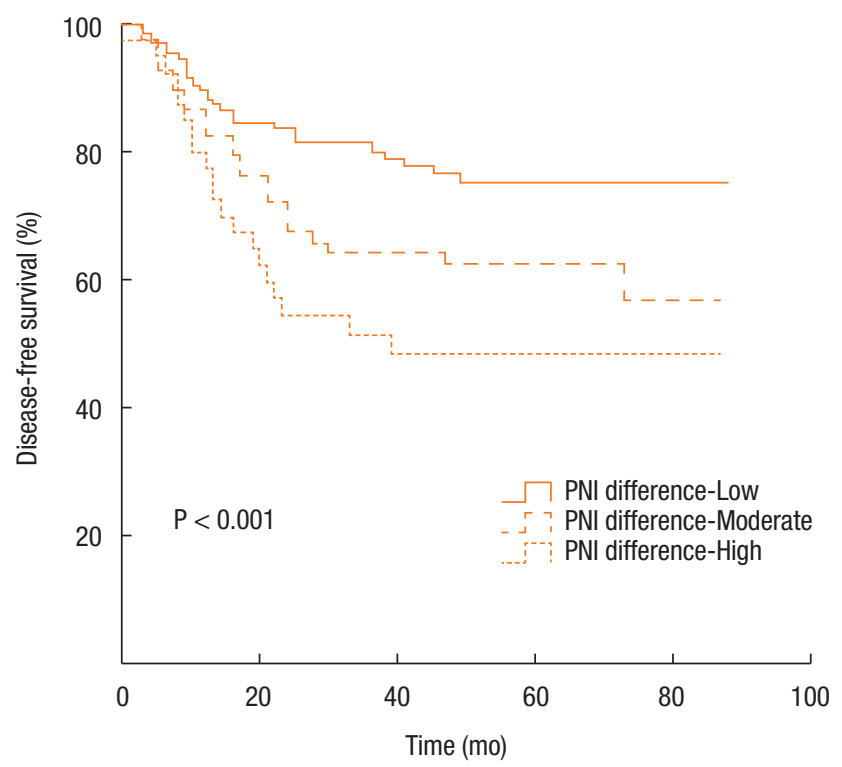

Fig. 3. Kaplan-Meier estimates and log-rank analyses of disease-free survival. PNI, prognostic nutritional index.

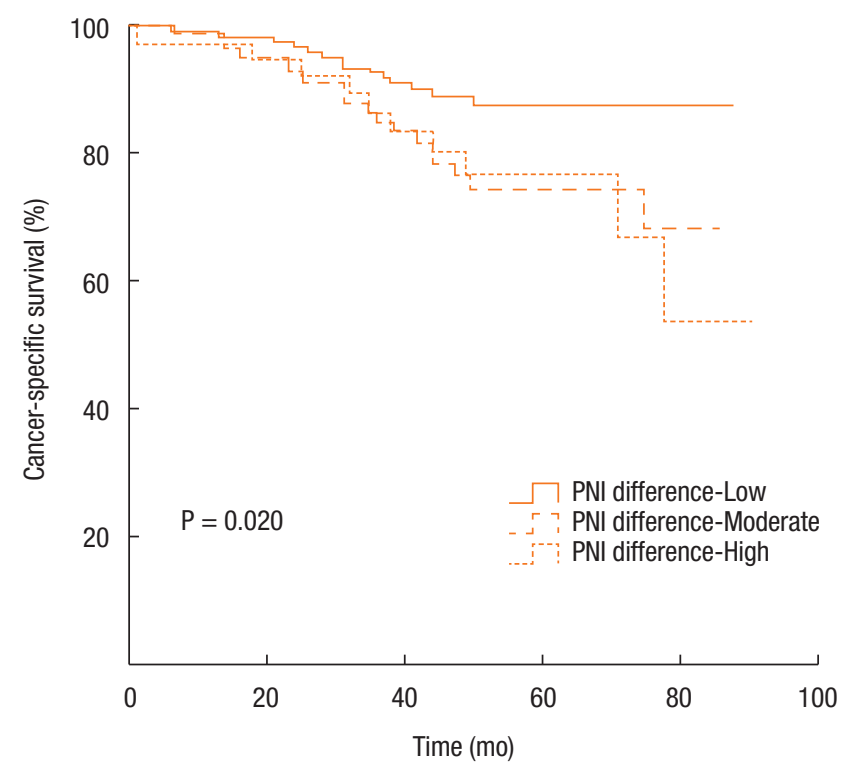

Fig. 4. Kaplan-Meier estimates and log-rank analyses of cancer-specific survival. PNI, prognostic nutritional index.

Table 2. Univariate and multivariate analyses of predictive factors for disease-free survival

\begin{tabular}{|c|c|c|c|c|}
\hline \multirow{2}{*}{ Factor } & \multirow{2}{*}{$\begin{array}{c}\text { Univariate analysis } \\
\text { P-value }\end{array}$} & \multicolumn{3}{|c|}{ Multivariate analysis } \\
\hline & & $\mathrm{HR}$ & $95 \% \mathrm{Cl}$ & P-value \\
\hline Age (<60 yr vs. $\geq 60$ yr) & 0.833 & 1.312 & $0.836-2.059$ & 0.237 \\
\hline Sex (male vs. female) & 0.979 & 0.861 & $0.528-1.404$ & 0.548 \\
\hline BMI $\left(<18.5\right.$ kg/m² vs. $\left.\geq 18.5 \mathrm{~kg} / \mathrm{m}^{2}\right)$ & 0.554 & 1.402 & $0.600-3.280$ & 0.435 \\
\hline Histology (WD/MD vs. PD/mucinous) & 0.007 & 1.583 & $0.871-2.877$ & 0.132 \\
\hline ypT stage (0/1/2 vs. $3 / 4)$ & 0.002 & 1.673 & $1.004-2.787$ & 0.048 \\
\hline ypN stage (0 vs. 1/2) & $<0.001$ & 2.378 & $1.494-3.786$ & $<0.001$ \\
\hline $\mathrm{PNI}(<5)$ & $<0.001$ & & & 0.002 \\
\hline vs. $5-10$ & & 1.705 & $1.027-2.831$ & 0.039 \\
\hline vs. $>10$ & & 2.792 & $1.577-4.942$ & $<0.001$ \\
\hline Complication (Dindo < III vs. $\geq$ III) & 0.490 & 0.786 & $0.282-2.191$ & 0.645 \\
\hline ASA score (I/II vs. III/IV) & 0.332 & 1.420 & $0.442-4.559$ & 0.556 \\
\hline AV distance (LOW vs. MID/UPPER) & 0.229 & 0.672 & $0.424-1.067$ & 0.092 \\
\hline CEA ( $<5 \mathrm{ng} / \mathrm{mL}$ vs. $\geq 5 \mathrm{ng} / \mathrm{mL})$ & 0.475 & 0.871 & $0.540-1.403$ & 0.569 \\
\hline
\end{tabular}

$\mathrm{HR}$, hazard ratio; $\mathrm{Cl}$, confidence interval; BMI, body mass index; WD, well differentiated; MD, moderately differentiated; PD, poorly differentiated; PNI, prognostic nutritional index; Dindo, Clavien-Dindo classification grade; ASA, American Society of Anesthesiologists; AV, anal verge; LOW, low rectal cancer, AV < 5 cm; MID/UPPER, middle/upper rectal cancer, $A V \geq 5 \mathrm{~cm}$; CEA, carcinoembryonic antigen.

studies of patients with colorectal cancer have shown that malnutrition is associated with a significant risk of wound complications and anastomosis site leakage $[3,5]$. Poor short-term postoperative outcomes might lead to inferior long-term oncologic outcomes [21]. However, the postoperative complication rates, including the overall and the high-grade complication rates, did not differ significantly among the 3 groups in our study.
Previous studies have shown a close correlation of inflammation with cancer progression [8]. In colorectal cancer, inflammatory markers such as the C-reactive protein level, neutrophil-to-lymphocyte ratio (NLR), Glasgow prognostic score (GPS), and modified GPS (mGPS) have been reported as prognostic markers [2224]. Notably, the serum albumin concentration reflects not only a patient's nutritional status, but also chronic inflammation. In ad- 
Table 3. Univariate and multivariate analyses of prognostic factors for cancer-specific survival

\begin{tabular}{|c|c|c|c|c|}
\hline \multirow{2}{*}{ Factor } & \multirow{2}{*}{$\frac{\text { Univariate analysis }}{\text { P-value }}$} & \multicolumn{3}{|c|}{ Multivariate analysis } \\
\hline & & HR & $95 \% \mathrm{Cl}$ & P-value \\
\hline Age (<60 yr vs. $\geq 60$ yr) & 0.307 & 1.679 & $0.906-3.109$ & 0.099 \\
\hline Sex (male vs. female) & 0.674 & 0.998 & $0.501-1.987$ & 0.996 \\
\hline BMI $(<18.5$ kg/m² vs. $\geq 18.5$ kg/m²) & 0.065 & 2.485 & $0.969-6.371$ & 0.058 \\
\hline Histology (WD/MD vs. PD/mucinous) & $<0.001$ & 3.189 & $1.622-6.269$ & 0.001 \\
\hline ypT stage (0/1/2 vs. 3/4) & 0.003 & 2.395 & $1.169-4.904$ & 0.017 \\
\hline ypN stage (0 vs. 1/2) & 0.029 & 1.337 & $0.702-2.549$ & 0.377 \\
\hline PNI $(<5)$ & 0.02 & & & 0.036 \\
\hline vs. $5-10$ & & 2.469 & $1.225-4.978$ & 0.012 \\
\hline vs. $>10$ & & 2.020 & $0.883-4.620$ & 0.096 \\
\hline Complication (Dindo < III vs. $\geq$ III) & 0.773 & 1.631 & $0.490-5.433$ & 0.426 \\
\hline ASA score (I/II vs. III/IV) & 0.301 & 1.211 & $0.266-5.513$ & 0.804 \\
\hline AV distance (LOW vs. MID/UPPER) & 0.866 & 0.950 & $0.500-1.804$ & 0.875 \\
\hline CEA (<5 ng/mL vs. $\geq 5$ ng/mL) & 0.498 & 0.901 & $0.469-1.731$ & 0.754 \\
\hline
\end{tabular}

$\mathrm{HR}$, hazard ratio; $\mathrm{Cl}$, confidence interval; $\mathrm{BMI}$, body mass index; WD, well differentiated; MD, moderately differentiated; PD, poorly differentiated; PNI, prognostic nutritional index; Dindo, Clavien-Dindo classification grade; ASA, American Society of Anesthesiologists; AV, anal verge; LOW, low rectal cancer, AV <5 cm; MID/UPPER, middle/upper rectal cancer, $A V \geq 5 \mathrm{~cm}$; CEA, carcinoembryonic antigen.

dition, the serum lymphocyte count has been identified as an objective parameter of inflammation. Although the PNI was first introduced to reflect the nutritional status of a cancer patient, the prognostic significance of this index can be attributed to its connection with the systemic inflammatory response.

Based on the findings of our study, patients' long-term outcomes might be enhanced by correcting their immunonutritional status during pCRT. Efforts to improve the outcomes of malnourished cancer patients by providing perioperative immunonutritional support are ongoing [8]. However, previous studies have included some limitations. Most studies included inhomogeneous patients with GI cancers of different origins; thus, the results were somewhat difficult to interpret $[4,7,8]$. Optimal timing of the immunonutritional intervention may be important. Generally, preoperative immunonutritional intervention is thought to be beneficial for patients undergoing GI surgery [25]. However, no available studies have discussed when to initiate and for how long to maintain an immunonutritional intervention for patients receiving pCRT for the treatment of rectal cancer. In the current study, we did not observe a significant intergroup difference with regard to tumor responses after pCRT or postoperative complications. Accordingly, such patients might not require immunonutritional intervention before pCRT initiation. The results of our study will lead to the design of a further prospective study. An enteral immunonutritional formula or parental nutritional formula could be provided upon admission to every patient undergoing PCRT, and the PNI or other scales, such as NRS-2002, could be used to assess improvements in the immunonutritional status. Validation of this enhancement of long-term oncologic outcomes in a pro- spective study could lead to new treatment options, of which preoperative immunonutritional support would be the treatment of choice.

The retrospective and single-center design of this study has some potential drawbacks. A risk exists for potential bias in patient selection and potential flaws in the accuracy of the documented medical records. Furthermore, potential confounding factors, such as albumin replacement therapy or infection, which could affect the serum albumin level and lymphocyte count, were not assessed. An additional weakness of this study is the small number of cases.

In patients with LARC, the dPNI during preoperative concurrent CRT is predictive of long-term outcomes. Our data suggest that correcting the patient's immunonutritional status during concurrent CRT could enhance oncologic outcomes. Such results warrant further investigation in the form of a prospective study.

\section{CONFLICT OF INTEREST}

No potential conflict of interest relevant to this article was reported.

\section{REFERENCES}

1. Salem ME, Hartley M, Unger K, Marshall JL. Neoadjuvant combined-modality therapy for locally advanced rectal cancer and its future direction. Oncology (Williston Park) 2016;30:546-62.

2. Glimelius B, Tiret E, Cervantes A, Arnold D; ESMO Guidelines Working Group. Rectal cancer: ESMO Clinical Practice Guide- 
lines for diagnosis, treatment and follow-up. Ann Oncol 2013;24 Suppl 6:vi81-8.

3. Schwegler I, von Holzen A, Gutzwiller JP, Schlumpf R, Mühlebach S, Stanga Z. Nutritional risk is a clinical predictor of postoperative mortality and morbidity in surgery for colorectal cancer. Br J Surg 2010;97:92-7.

4. Beattie AH, Prach AT, Baxter JP, Pennington CR. A randomised controlled trial evaluating the use of enteral nutritional supplements postoperatively in malnourished surgical patients. Gut 2000;46:813-8.

5. Kwag SJ, Kim JG, Kang WK, Lee JK, Oh ST. The nutritional risk is a independent factor for postoperative morbidity in surgery for colorectal cancer. Ann Surg Treat Res 2014;86:206-11.

6. Jie B, Jiang ZM, Nolan MT, Zhu SN, Yu K, Kondrup J. Impact of preoperative nutritional support on clinical outcome in abdominal surgical patients at nutritional risk. Nutrition 2012;28:1022-7.

7. Xu J, Zhong Y, Jing D, Wu Z. Preoperative enteral immunonutrition improves postoperative outcome in patients with gastrointestinal cancer. World J Surg 2006;30:1284-9.

8. Burden S, Todd C, Hill J, Lal S. Pre-operative nutrition support in patients undergoing gastrointestinal surgery. Cochrane Database Syst Rev 2012;11:CD008879.

9. Braga M, Gianotti L, Vignali A, Carlo VD. Preoperative oral arginine and n-3 fatty acid supplementation improves the immunometabolic host response and outcome after colorectal resection for cancer. Surgery 2002;132:805-14.

10. Goseki N, Okamoto A, Onodera T. Postoperative nutritional assessment in gastric and colorectal cancer. Nihon Geka Gakkai Zasshi 1986;87:853-8.

11. Migita K, Takayama T, Saeki K, Matsumoto S, Wakatsuki K, Enomoto $\mathrm{K}$, et al. The prognostic nutritional index predicts longterm outcomes of gastric cancer patients independent of tumor stage. Ann Surg Oncol 2013;20:2647-54.

12. Jiang N, Deng JY, Ding XW, Ke B, Liu N, Zhang RP, et al. Prognostic nutritional index predicts postoperative complications and long-term outcomes of gastric cancer. World J Gastroenterol 2014;20:10537-44.

13. Mori S, Usami N, Fukumoto K, Mizuno T, Kuroda H, Sakakura N, et al. The significance of the prognostic nutritional index in patients with completely resected non-small cell lung cancer. PLoS One 2015;10:e0136897.

14. Mohri Y, Inoue Y, Tanaka K, Hiro J, Uchida K, Kusunoki M.
Prognostic nutritional index predicts postoperative outcome in colorectal cancer. World J Surg 2013;37:2688-92.

15. Yang Y, Gao P, Chen X, Song Y, Shi J, Zhao J, et al. Prognostic significance of preoperative prognostic nutritional index in colorectal cancer: results from a retrospective cohort study and a metaanalysis. Oncotarget 2016;36:58543-52.

16. Jian-Hui C, Iskandar EA, Cai ShI, Chen CQ, Wu H, Xu JB, et al. Significance of Onodera's prognostic nutritional index in patients with colorectal cancer: a large cohort study in a single Chinese institution. Tumour Biol 2016;37:3277-83.

17. Sun K, Chen S, Xu J, Li G, He Y. The prognostic significance of the prognostic nutritional index in cancer: a systematic review and meta-analysis. J Cancer Res Clin Oncol 2014;140:1537-49.

18. Dindo D, Demartines N, Clavien PA. Classification of surgical complications: a new proposal with evaluation in a cohort of 6336 patients and results of a survey. Ann Surg 2004;240:205-13.

19. Onodera T, Goseki N, Kosaki G. Prognostic nutritional index in gastrointestinal surgery of malnourished cancer patients. Nihon Geka Gakkai Zasshi 1984;85:1001-5.

20. Mandard AM, Dalibard F, Mandard JC, Marnay J, Henry-Amar $\mathrm{M}$, Petiot JF, et al. Pathologic assessment of tumor regression after preoperative chemoradiotherapy of esophageal carcinoma. Clinicopathologic correlations. Cancer 1994;73:2680-6.

21. McSorley ST, Horgan PG, McMillan DC. The impact of the type and severity of postoperative complications on long-term outcomes following surgery for colorectal cancer: a systematic review and meta-analysis. Crit Rev Oncol Hematol 2016;97:168-77.

22. Toiyama Y, Inoue Y, Saigusa S, Kawamura M, Kawamoto A, Okugawa $\mathrm{Y}$, et al. C-reactive protein as predictor of recurrence in patients with rectal cancer undergoing chemoradiotherapy followed by surgery. Anticancer Res 2013;33:5065-74.

23. Ishizuka M, Nagata H, Takagi K, Iwasaki Y, Kubota K. Inflammation-based prognostic system predicts postoperative survival of colorectal cancer patients with a normal preoperative serum level of carcinoembryonic antigen. Ann Surg Oncol 2012;19:3422-31.

24. Park BK, Park JW, Han EC, Ryoo SB, Han SW, Kim TY, et al. Systemic inflammatory markers as prognostic factors in stage IIA colorectal cancer. J Surg Oncol 2016;114:216-21.

25. Mazaki T, Ishii Y, Murai I. Immunoenhancing enteral and parenteral nutrition for gastrointestinal surgery: a multiple-treatments meta-analysis. Ann Surg 2015;261:662-9. 